\title{
An open, comparative, multicentre clinical study of combined oral therapy with sildenafil and doxazosin GITS for treating Chinese patients with erectile dysfunction and lower urinary tract symptoms secondary to benign prostatic hyperplasia
}

\author{
Zhe Jin ${ }^{1}$, Zhi-Chao Zhang ${ }^{1}$, Ji-Hong Liu ${ }^{2}$, Jun Lu ${ }^{3}$, Yu-Xin Tang ${ }^{4}$, Xiang-Zhou Sun ${ }^{5}$, Wei-Dong Song ${ }^{1}$, Bing Gao $^{1}$, \\ Ying-Lu Guo ${ }^{1}$ and Zhong-Cheng Xin ${ }^{1}$
}

This study sought to investigate the clinical efficacy and safety of combined oral therapy with sildenafil and doxazosin GITS compared to sildenafil monotherapy in treating Chinese patients with erectile dysfunction (ED) and lower urinary tract symptoms secondary to benign prostatic hyperplasia (BPH/LUTS). The trial was conducted in hospitals in Beijing, Shanghai, Changsha, Wuhan and Guangzhou, five major cities in China. A total of 250 patients diagnosed with ED and BPH/LUTS aged 50-75 years, and who had International Index of Erection Function-5 (IIEF-5) scores $\leqslant 21$ and International Prostate Symptom Score (IPSS) $\geqslant 10$ points, were enrolled and randomly divided into Group A (168 cases; doxazosin GITS $4 \mathrm{mg}$ once daily plus sildenafil 25-100 mg on demand) and Group B ( 82 cases; sildenafil 25-100 mg on demand). Efficacies were evaluated by IIEF-5 and IPSS scores and a quality of life (QoL) questionnaire, and adverse effects were evaluated during the treatment period. There were no statistically significant differences in mean age, and IIEF-5, IPSS and QoL scores pre-treatment between the two groups. After treatment, IIEF-5, IPSS and QoL scores were significantly improved in Group A, while only IIEF-5 scores were significantly improved in Group B compared with pre-treatment. There were no significant differences in side effects between the two groups. The results indicated that combined therapy with sildenafil and doxazosin GITS for the treatment of ED and BPH/LUTS is safe and effective compared to sildenafil monotherapy.

Asian Journal of Andrology (2011) 13, 630-635; doi:10.1038/aja.2010.177; published online 23 May 2011

Keywords: doxazosin GITS; erectile dysfunction; IPSS; lower urinary tract symptoms; quality of life; sildenafil

\section{INTRODUCTION}

With increasing human life expectancies, age-related diseases have become an important clinical issue. The medical profession will be faced with the task of ensuring that the ageing population remains healthy and maintains a good quality of life (QoL). From the results of many epidemiological studies, we know that erectile dysfunction (ED), benign prostatic hyperplasia $(\mathrm{BPH})$, cardiovascular disease and depression are the four major non-cancerous diseases in males over 50 years old, and the morbidity of those diseases increases with advancing age. ${ }^{1}$ The rates of $\mathrm{ED}$ and $\mathrm{BPH}$ in men over 40 years of age are $52 \%$ and $56 \%$, respectively. Approximately half of men over 60 years old suffer from $\mathrm{BPH}$, and $15 \%$ $30 \%$ of these men have lower urinary tract symptoms (LUTS). ${ }^{2,3}$ Studies have indicated that LUT severity may be related to ED prevalence. ${ }^{4}$ These studies have indicated that $\mathrm{ED}$ and $\mathrm{BPH}$ coexist in ageing males at a high rate, and that the combination of these conditions seriously interferes with daily life, decreasing the QoL of ageing men. BPH can have a profound effect on a patient's QoL and sexual function. However, little clinical research has focused on ED combined with BPH /LUTS. ${ }^{5}$

Although the mechanism of ED and BPH/LUTS remain to be investigated, some of studies have been reported that smooth muscle dysfunction in prostrate and/or penis may induce dysuria and/or ED due to increased Rho-kinase channel activity, which may be related to further constriction of the smooth muscle and decrease in nitric oxide synthese activity and utility affects $\mathrm{NO}-$ cyclic guanosine monophosphate signalling channels, altering endothelial cell function and leading to arteriosclerosis, autonomic nervous system overactivity and disturbance, ${ }^{6}$ and endothelial nitric oxide synthase $894 \mathrm{~T}$ allele gene susceptive may have relationship to ED and BPH/LUTS. ${ }^{7}$

We hypothesize that combined oral therapy with sildenafil and doxazosin will be more effective than sildenafil alone for improving erectile function and LUTS suggestive of $\mathrm{BPH}$, thus improving QoL in patients with ED and BPH/LUTS.

\footnotetext{
${ }^{1}$ Andrology Center, Peking University First Hospital, Peking University, Beijing 100034, China; ${ }^{2}$ Department of Urology, Tongji Hospital, Tongji Medical College, Huazhong University of Science and Technology, Wuhan 430030, China; ${ }^{3}$ Departement of Urology, The First Affiliated Hospital, Shanghai Jiao Tong University, Shanghai 200080, China; ${ }^{4}$ Department of Urology, The Third Xiangya Hospital of Central South University, Changsha, Hunan 410013, China and ${ }^{5}$ Department of Urology, The First Affiliated Hospital of Sun Yat-Sen University, Guangzhou 510080, China

Correspondence: Professor ZC Xin (xinzc@bjmu.edu.cn, xinzc08@gmail.com)

Received: 12 November 2010; Revised: 4 December 2010; Accepted: 10 December 2010; Published online: 23 May 2011
} 


\section{MATERIALS AND METHODS}

\section{Patient enrolment}

The trial was conducted in five centres of medical university-associated hospitals located in Beijing (northern China), Shanghai (eastern China), Changsha (southern China), Wuhan (central China) and Guangzhou (southeastern China) from September 2007 to October 2009. Approvals were obtained from local ethics committees of each hospital. All patients gave written informed consent prior to the study. A total of 250 patients aged 50-75 years who were diagnosed ED and $\mathrm{BPH} / \mathrm{LUTS}$ were enrolled in this study and 203 patients $(81.2 \%)$ completed the entire follow-up evaluation.

Patients were recruited for the trial according to their International Index of Erection Function-5 scores (IIEF- $5 \leqslant 21$ ) and International Prostate Symptom Scores (IPSS $\geqslant 10$ points). Patients who had no medical, surgical or experimental interventions for BPH previous 2 weeks to the study were eligible to participate. Other inclusion criteria included a maximum urinary flow rate $(\mathrm{Qmax}) \leqslant 15 \mathrm{ml} \mathrm{s}^{-1}$ for a voided volume $\geqslant 150 \mathrm{ml}$ with a post-voiding volume $<150 \mathrm{ml}$, a prostate volume $\geqslant 25 \mathrm{ml}$ and a serum prostate-specific antigen level $<4 \mathrm{ng} \mathrm{ml}^{-1}$. All patients were heterosexual with stable partners. A normal blood testosterone level was also used as an inclusion criterion.

Exclusion criteria included patients with significant cardiovascular disease, uncontrolled diabetes mellitus, neurological and psychiatric disorders, previous genitourinary surgery, and history of hypersensitivity and contraindication to one of the study drugs or participation in another clinical trial in 3 months prior to the study.

\section{Study design}

This study was a two-armed, randomized, parallel, non-inferior, comparative study of doxazosin (Cardura XL; Pfizer Inc., Dalian, China) $4 \mathrm{mg}$ once daily plus sildenafil (Viagra; Pfizer Inc.) $25-100 \mathrm{mg}$ on demand compared with sildenafil monotherapy in the treatment of patients with ED and BPH/LUTS. No placebo arm was included because of the difficulty of placebo preparation.

Patients who fulfilled the entry criteria at selection were randomized to combined-therapy group (Group A, $n=168$ ) or monotherapy group (Group B, $n=82$ ). In Group A, patients received doxazosin GITS ( $4 \mathrm{mg} \mathrm{d}^{-1}$ ) plus sildenafil (25-100 $\mathrm{mg}$ on demand) with at least 6-h interval between the administrations of the two drugs, while in Group B, patients only received sildenafil treatment (25-100 mg on demand). According to the IIEF-5 score, each group was divided into subgroups for mild (17-21), moderate (12-16) and severe (1-11) ED. According to the IPSS score, each group was divided into subgroups for moderate $(10-19)$ and severe $(\geqslant 20)$.

Serum prostate-specific antigen, testosterone and uroflowmetry testing were also conducted during the screening period. Clinical efficacies assessments for ED and BPH/LUTS were evaluated by IIEF-5, IPSS and QoL questionnaires, and follow-up assessments were performed by telephone inquiries and email communication. Safety profiles were assessed by physical examination (heart rate and blood pressure), laboratory tests and monitoring clinical adverse events.

\section{Assessment}

Clinical efficacy assessments for ED and BPH/LUTS were evaluated by IIEF-5, IPSS and QoL. The results of IIEF-5, IPSS and QoL were used to evaluate related symptoms at pre-treatment (Pre Tx), 3 and 6 months post-treatment in both groups. The IPSS was used to assess LUTS and is the internationally accepted standard questionnaire for assessing BPH/LUTS. It is a validated, seven-item scale that assesses the severity of incomplete emptying, urinary frequency, intermittency, urgency, weak stream and nocturia. The seven items have an ordered categorical response that can be scored from 0 to 5, with an overall score of $0-35$. The severity of symptoms was classified as moderate $(10-19)$ or severe $(\geqslant 20)$.

Sexual function was assessed using the IIEF-5. The IIEF-5 questionnaire (also referred to as the Sexual Health Inventory of Men) is an abridged, five-item version of the IIEF-15 questionnaire. It is a validated, multidimensional questionnaire that is a sensitive indicator of changes in erectile function and treatment outcomes. It is scored from 1 to 5 . The total IIEF- 5 score, calculated by totalling the responses to all five questions, is interpreted as follows: normal (22-25); mild ED (17-21); moderate ED (12-16); and severe ED (1-11). Safety is assessed as the incidence of adverse events. ${ }^{8}$

QoL was assessed using QoL scores to evaluate the degree of negative impact by BPH/LUTS.

\section{Statistical analysis}

Data are expressed as mean \pm s.d. A statistical difference analysis was carried out by a chi-square test, analysis of covariance and independent and paired $t$-test. All analyses were two-tailed, with a significance level of 5\%. Analyses were performed using the SPSS 13.0 software package (SPSS Inc., Chicago, IL, USA).

\section{RESULTS}

\section{Patient characteristics}

Among the 250 cases enrolled, 221 (88.4\%) completed the 3-month follow-up evaluation (Group A: 149/168; Group B: 72/82), and 203 patients $(81.2 \%)$ finished the 6-month follow-up evaluation (Group A: 137/168; Group B: 66/82). The average patient age and body mass index for each group and the subgroup ratio of ED and BPH/LUTS severity between the two groups were not significantly different ( $P=0.315, P=0.441, P=0.535$ and $P=0.431$, respectively). The Qmax and post-void residual (PVR) also showed no significant differences between the two groups ( $P=0.074$ and $P=0.181$, respectively) (Table 1).

\section{IIEF-5 total score changes}

In Group A, the Pre Tx, 3-month, 6-month scores post-treatment were $13.0 \pm 3.0,18.4 \pm 4.4$ and 19.9 \pm 4.4 , and in Group B, they were $12.4 \pm 3.6,16.8 \pm 5.6$ and 17.2 \pm 5.7 , respectively. IIEF-5 total scores evaluated at Pre Tx showed no significant difference between the two groups $(P=0.202)$. IIEF-5 total scores were significant increases in the 3- and 6-month post-treatment compared to the Pre Tx (all $P=0.000)$ in both groups. Score changed in time-dependent manner $(P=0.0001)$. IIEF-5 scores, as evaluated at 3 and 6 months postmedication in Group A, were markedly improved compared to those in Group B ( $P=0.022$ and $P=0.0001$, respectively) (Figure 1a).

\section{IIEF-5 score changes in subgroups}

In both Group A and Group B, all mild, moderate and severe ED patients showed significant improvements in IIEF-5 scores at 3 - and 6-month post-medication compared to pre-medication (all $P=0.000$ in Group A and all $P<0.05$ in Group B). In IIEF- 5 scores between two groups at the end of 3-month medication, there was a significant difference for severe ED patients $(P=0.008)$ but not for mild or moderate ED patients ( $P=0.875$ and $P=0.081$, respectively). At the end of 6-month medication, there was a significant difference for severe and moderate ED patients ( $P=0.001$ and $P=0.000$, respectively) but not for mild ED patients $(P=0.959)$ (Figure $\mathbf{1 b}-\mathbf{d}$ ). 
Table 1 Patients' characteristics (total 250 who were enrolled)

\begin{tabular}{lccc}
\hline & $\begin{array}{c}\text { Group } A \\
(\mathrm{n}=168)\end{array}$ & $\begin{array}{c}\text { Group B } \\
(\mathrm{n}=82)\end{array}$ & P value \\
\hline Hospital location & $55(32.7)$ & $24(29.3)$ & \\
$\quad$ Beijing, $n(\%)$ & $26(15.5)$ & $12(14.6)$ & \\
Shanghai, $(n$ (\%) & $20(11.9)$ & $10(12.2)$ & \\
$\quad$ Changsha, $n(\%)$ & $35(20.8)$ & $16(19.5)$ & \\
$\quad$ Wuhan, $n(\%)$ & $32(19.1)$ & $20(24.4)$ & \\
$\quad$ Guangzhou, $n(\%)$ & & & \\
Age (years) & $61.7 \pm 5.3$ & $60.8 \pm 5.9$ & 0.315 \\
Body mass index (kg m $\left.{ }^{-2}\right)$ & $23.5 \pm 3.9$ & $24.0 \pm 3.5$ & 0.441 \\
Qmax (ml s ${ }^{-1}$ ) & $10.7 \pm 2.1$ & $11.2 \pm 1.3$ & 0.074 \\
PVR (ml) & $23.0 \pm 14.1$ & $20.5 \pm 11.3$ & 0.181 \\
BPH/LUTS severity & & & \\
$\quad$ Moderate (IPSS 10-19), $n(\%)$ & $102(60.8)$ & $54(65.8)$ & 0.431 \\
$\quad$ Severe (IPSS $\geqslant 20), n(\%)$ & $66(39.2)$ & $28(34.2)$ & \\
ED severity & & & \\
$\quad$ Mild (IIEF-5 17-21), $n(\%)$ & $13(7.7)$ & $8(9.7)$ & 0.535 \\
$\quad$ Moderate (IIEF-5 12-16), $n(\%)$ & $126(75.0)$ & $56(68.3)$ & \\
$\quad$ Severe (IIEF-5 5-11), $n(\%)$ & $29(17.3)$ & $18(22.0)$ & \\
\hline
\end{tabular}

Abbreviations: BPH/LUTS, lower urinary tract symptoms secondary to benign prostatic hyperplasia; ED, erectile dysfunction; IIEF-5, International Index of Erection Function-5; IPSS, International Prostate Symptom Score; PVR, post-void residual; Qmax, a maximum urinary flow rate.

Group A, sildenafil and doxazosin GITS combined therapy. Group B, sildenafil monotherapy. Values are expressed as mean \pm s.d. unless stated otherwise. Independent and paired $t$-test was carried out for the agents of age, body mass index, Qmax and PVR. Chi-square test was carried out for the agent of ED and BPH/LUTS severity.
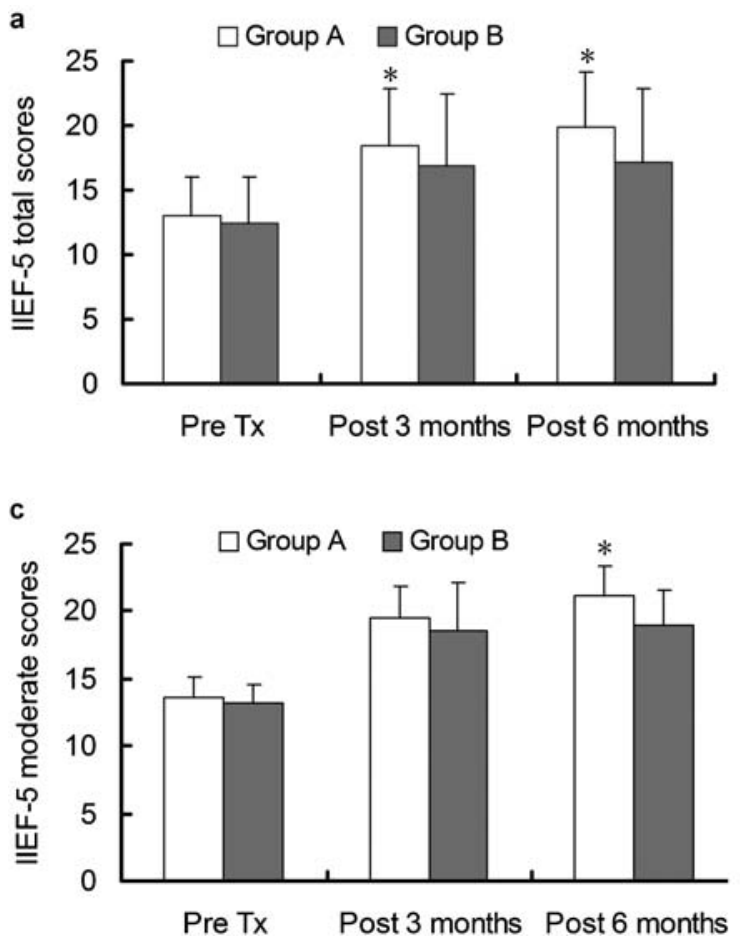

IPSS total score changes

In Group A, the pre-medication, 3- and 6-month post-medication IPSS scores were 18.4 $\pm 5.8,13.6 \pm 5.8$ and 10.2 \pm 5.4 , and in Group B, they were $16.9 \pm 4.5,16.8 \pm 5.3$ and $17.2 \pm 5.8$, respectively. The Pre Tx IPSS total scores did not differ significantly between the two treatment groups $(P=0.080)$. In Group $\mathrm{A}$, the 3 -month post-treatment score was significantly reduced compared to the pre-medication $(P<0.05)$, and the 6-month score post-treatment was also reduced compared to the 3month score $(P<0.05)$. Compared to the pre-medication score, there were no marked changes in the 3 - and 6-month post-treatment scores ( $P=0.385$ and $P=0.233$, respectively) in Group B. Significant improvements in Group A versus Group B were also observed at both 3 and 6 months post-treatment $(P<0.05)$. (Figure $2 \mathbf{a})$.

\section{IPSS score changes in subgroups}

When the IPSS total score was separated into moderate (10-19) and severe $(\geqslant 20)$ subgroups, in Group A, there was a significant reduction among the Pre Tx, 3- and 6-month post-treatment scores for moderate and severe patients (all $P=0.000$ ). In Group B, there were no significant differences among the Pre Tx, 3- and 6-month post-treatment scores (all $P>0.05$ ) for moderate patients, and in severe patients, both the 3- and 6-month post-treatment scores increased, and at 3-month post-treatment evaluation, there was no significant difference $(P=0.076)$, but after 6 months post-treatment, the symptoms were markedly worse $(P=0.013)$. In moderate patients (IPSS 10-19), significant improvements with Group A versus Group B were observed at both 3 and 6 months post-treatment (Figure 2b). In severe patients
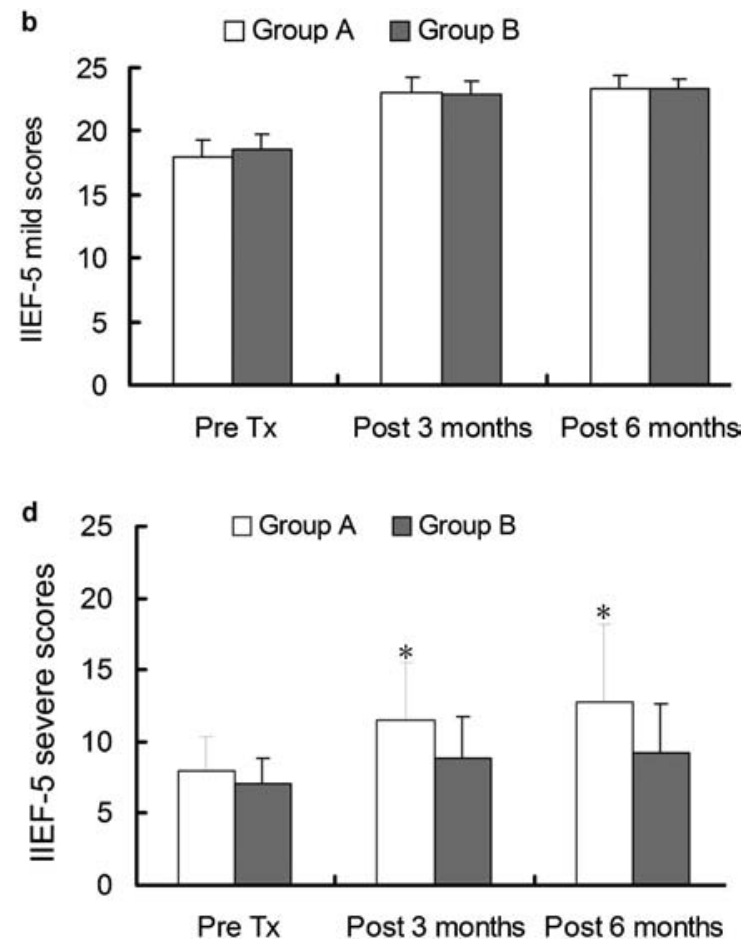

Figure 1 (a) IIEF-5 total score changes over time. Significant improvements with sildenafil and doxazosin GITS combined therapy (Group A) versus sildenafil monotherapy (Group B) were observed both at 3 and 6 months post-medication. (b) IIEF-5 mild score comparison between the two groups. In mild ED patients (IIEF-5: 17-21), no significant improvements with combined therapy versus monotherapy were observed both at 3 and 6 months post-medication (P>0.05). (c) IIEF-5 moderate score comparison between the two groups. In moderate ED patients (IIEF-5: 12-16), significant improvements with combined therapy versus monotherapy were observed at 6 months $(P=0.000)$ post-medication but not at 3 months $(P>0.05)$. (d) Severe ED patients IIEF-5 score comparison between the two groups. In severe ED patients (IIEF-5: 5-11), significant improvements with combined therapy versus monotherapy were observed both at 3 months $(P=0.008)$ and 6 months $(P=0.001)$ post-medication. $* P<0.05$, compared with Group B. Statistical difference analysis was carried out by analysis of variance of repeated data measured. ED, erectile dysfunction; IIEF-5, International Index of Erection Function-5; Pre Tx, pre-treatment. 

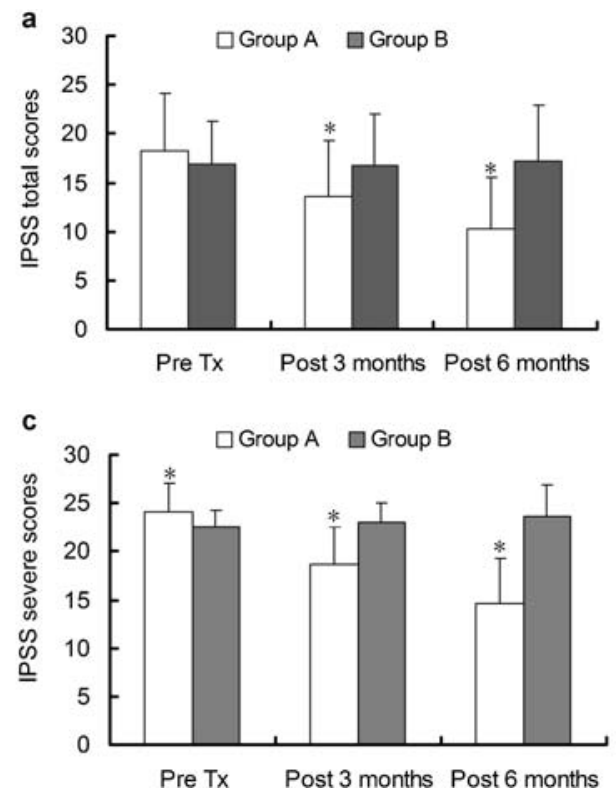
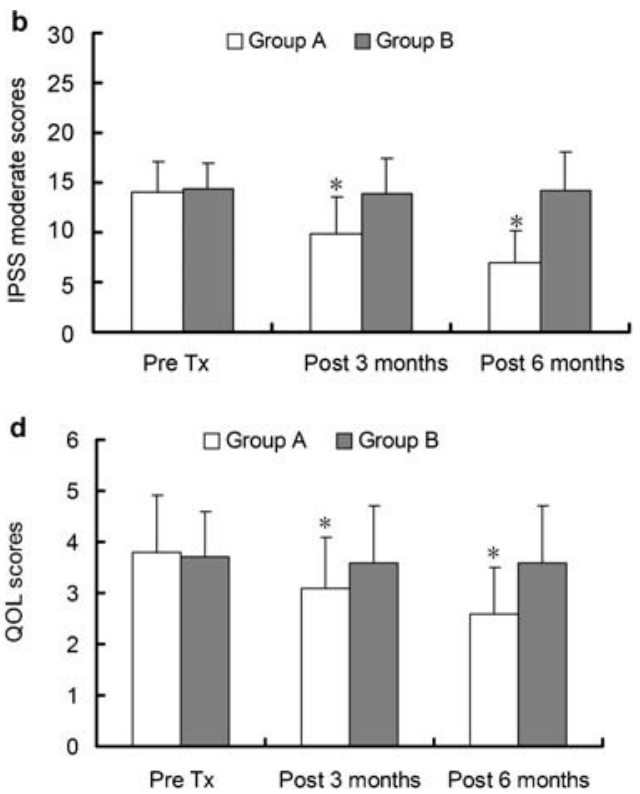

Figure 2 (a) IPSS total score changes over time. Significant improvements with sildenafil and doxazosin GITS combined therapy (Group A) versus sildenafil monotherapy (Group B) were observed both at 3 and 6 months post-medication. (b) Moderate BPH/LUTS patients' IPSS score comparison between the two groups. In moderate BPH/ LUTS patients (IPSS: 10-19), significant improvements with Group A versus Group B were observed both at 3 and 6 months post-medication. (c) Severe BPH/LUTS patients' IPSS score comparison between the two groups. In severe BPH/LUTS patients (IPSS $\geqslant 20$ ), significant improvements with Group A versus Group B were observed at pre-medication, 3 and 6 months post-medication. (d) QoL total score changes over time. Significant improvements with Group A versus Group B were observed both at 3 and 6 months post-medication. $* P<0.05$, compared with Group B. BPH/LUTS, lower urinary tract symptoms secondary to benign prostatic hyperplasia; IPSS, International Prostate Symptom Score; Pre Tx, pre-treatment; QoL, quality of life.

(IPSS $\geqslant 20$ ), significant improvements with Group A versus Group B were observed at Pre Tx, 3 and 6 months post-treatment (Figure 2c).

\section{QoL score changes}

QoL total scores Pre Tx showed no significantly difference between the two treatment groups. In Group A, the 3- and 6-month post-treatment scores were significantly reduced compared to Pre Tx score (both $P=0.000)$. In Group $\mathrm{B}$, there was no significant difference in the 3- and 6-month post-treatment scores compared to Pre Tx (both $P>0.05)$. Patients' life quality was improved with QoL total scores being significantly decreased in Group A compared to that in Group B at both 3 and 6 months post-treatment (Figure 2d).

\section{Sexual intercourse changes}

At the end of 6-month therapy, the percentage of patients who performed in sexual intercourse more than four times per month was $64.2 \%$ (Group A) and 60.6\% (Group B); there was no significant difference between the two groups $(P=0.616)$, but the percentage for each group was significantly increased compared to the Pre Tx (Group A, 30.1\%; Group B, 28.9\%; both $P=0.000$ ).

Increases in the frequency of penetration (IIEF-5, Q3) and maintained erections (IIEF-5, Q4) were greater in Group A (Q3+Q4 $\geqslant 8$, $60.2 \%)$ than in Group B (Q3+Q4 $\geqslant 8,42.3 \%)(P=0.011)$.

\section{Safety}

The number of patients who complained about adverse effects at the end of 6 months of therapy were $26(15.4 \%)$ in Group A and 12 $(14.6 \%)$ for Group B $(P>0.05)$, and some patients complained two or more kinds of adverse effects either in Group A or in Group B (Table 2). However, all symptoms were mild, and no participant left the study owing to adverse symptoms. The average heart rate and systolic and diastolic pressures at Pre Tx were $74.7 \pm 5.3 \mathrm{bpm}$, $123.8 \pm 9.0 \mathrm{mmHg}$ and $71.2 \pm 6.8 \mathrm{mmHg}$, respectively, in Group A, and $74.6 \pm 5.2 \mathrm{bpm}, 118.0 \pm 11.5 \mathrm{mmHg}$ and $70.4 \pm 7.1 \mathrm{mmHg}$, respectively, in Group B. At the end of 3-month mediation, the values of Group A were $75.2 \pm 4.8 \mathrm{bpm}, 124.0 \pm 9.1 \mathrm{mmHg}$ and $71.1 \pm 6.7 \mathrm{mmHg}$ (compared to Pre Tx, $P=0.353,0.057$ and 0.863 , respectively) and in Group $B$, the values were $74.0 \pm 5.1 \mathrm{bpm}$, $117.8 \pm 11.3 \mathrm{mmHg}$ and $70.2 \pm 7.3 \mathrm{mmHg}$ (compared to Pre Tx, $P=0.434,0.103$ and 0.787 , respectively). At the end of 6-month treatment, the values in Group A were $75.7 \pm 4.9 \mathrm{bpm}, 123.9 \pm 9.1 \mathrm{mmHg}$ and 70.9 $\pm 6.6 \mathrm{mmHg}$ (compared to Pre Tx, $P=0.087,0.198$ and 0.390 , respectively) and in group $B$, they were $75.0 \pm 5.8 \mathrm{bpm}$, $119.2 \pm 11.7 \mathrm{mmHg}$ and $69.9 \pm 7.0 \mathrm{mmHg}$, respectively (compared to Pre Tx, $P=0.725,0.083$ and 0.349 , respectively), and there was no statistical significance. Mean laboratory parameters fell within normal

Table 2 Treatment-emergent adverse events during 6-months medication

\begin{tabular}{lcc}
\hline Adverse event & Group A $(\mathrm{n}=168)$ & Group B $(\mathrm{n}=82)$ \\
\hline Headache, $n(\%)$ & $9(5.4)$ & $5(6.1)$ \\
Dizzy, $n(\%)$ & $8(4.8)$ & $4(4.9)$ \\
Facial flushing, $n(\%)$ & $6(3.6)$ & $4(4.9)$ \\
Palpitation, $n(\%)$ & $6(3.6)$ & $2(2.4)$ \\
Dyspepsia, $n(\%)$ & $5(3.0)$ & $2(2.4)$ \\
Diarrhea, $n(\%)$ & $4(2.4)$ & $1(1.2)$ \\
Acratia, $n(\%)$ & $2(1.2)$ & $1(1.2)$ \\
Abdominal pain, $n(\%)$ & $1(0.6)$ & $0(0)$
\end{tabular}

Group A, sildenafil and doxazosin GITS combined therapy. Group B, sildenafil monotherapy. Values $(n)$ shown are the numbers of patients affected $(\%)$. 
ranges at all times, and changes in these parameters during the study period were minor and not considered clinically relevant.

\section{DISCUSSION}

$\mathrm{BPH}$ and $\mathrm{ED}$ are common diseases in ageing males. LUTS are common in patients with $\mathrm{BPH}$, and the probability of $\mathrm{BPH}$ occurring concurrently with $\mathrm{ED}$ is high. International epidemiological studies have shown that there is a strong relationship between LUTS and erectile dysfunction, and it is not influenced by age or comorbidities. ${ }^{9,10}$ Phosphodiesterase type 5 (PDE5) inhibitors (e.g., sildenafil) are first-line medications for ED therapy, and $\alpha 1$-adrenergic receptor inhibitors (e.g., doxazosin) have been shown to be effective in treating BPH/LUTS; close observation of pathogenically conditions and medication-based treatments have been the first-line therapy for BPH/ LUTS. $^{11}$

A basic research study found that PDE4 and PDE5 are located in the prostate gland. ${ }^{12}$ The results of this study gave rise to the speculation that drugs such as PDE4 and PDE5 inhibitors, which elevate the intracellular levels of cyclic adenosine monophosphate and cyclic guanosine monophosphate, may influence smooth muscle tone; therefore, doxazosin reduces adrenergic tension and may increase the level of cyclic adenosine monophosphate in smooth muscle of corpus cavernosum to facilitate erectile function.

Kaplan et al. ${ }^{13}$ reported that after treatment with alfuzosin $(10 \mathrm{mg}$ once daily), sildenafil ( $25 \mathrm{mg}$ once daily) or a combination of the two drugs for 12 weeks in patients with previously untreated LUTS and $\mathrm{ED}$, improvements in IPSS were significant for all three treatments, but were the greatest for the combined therapy. Frequency, nocturia, PVR and Qmax were significantly improved with alfuzosin alone and with the combined treatment. Improvements in IIEF were significant for sildenafil alone, and greater with the combined treatment, but there was no significant for alfuzosin alone. Likewise, increases in the frequency of penetration and maintained erections were greater in the combination therapy than alfuzosin or sildenafil alone. ${ }^{13}$ These investigations demonstrated that the combined use of a PDE5 inhibitor and an $\alpha 1$-adrenergic receptor inhibitor might be more effective than monotherapy with either agent.

As effective medications for ED and BPH therapy, the combination therapy of doxazosin and sildenafil for refractory ED showed greater effectiveness than sildenafil alone. This finding correlates with the anti-adrenergic function of doxazosin, which decreases the tension of the sympathetic nervous system and facilitates the effect of sildenafil stimulating the non-adrenergic non-cholinergic nervous system, augmenting penile erectile function. Oger et al. ${ }^{14}$ reported that in both cavernosal and prostatic tissues, the combination of sildenafil and doxazosin exerted a greater relaxant effect on concentration-response curves of phenylephrine or norepinephrine (NE) compared to either compound alone. Sildenafil significantly enhanced the relaxant effect of doxazosin on electrical field stimulation-induced contractions in prostatic strips. Doxazosin significantly increased the ability of sildenafil to inhibit NE-induced contractions in cavernosal strips. And in vitro, a PDE5 inhibitor (tadalafil) significantly inhibited NEinduced prostatic strip contractions by reducing the maximal effect, whereas $\alpha 1$-blockers (alfuzosin) shifted concentration-response curves of NE to the right, and the combination of both drugs resulted in a greater relaxant effect. ${ }^{15}$

In our study, the average age, body mass index and subgroup ratios of patients in both groups showed no significant differences; therefore, we can eliminate the effects of age, medication absorption, distribution and disease severity on the observed efficacy. In addition, there was little difference in the total Pre Tx IPSS, QoL, IIEF-5, Qmax and PVR scores, indicating that the Pre Tx severity of ED, LUTS secondary to $\mathrm{BPH}$ and urination effects on QoL was similar for the two groups.

In our study, sildenafil shows marked efficacy on ED; when combined with doxazosin, it had more significantly improved IIEF-5 score. With increasing treatment time, both groups showed better improvements in IIEF-5 score, which may be associated with increases in the frequency of sexual intercourse. At the end of 6 months of therapy, the percentage of patients who had intercourse more than four times a month was $64.2 \%$ (combined-therapy group) and $60.6 \%$ (monotherapy group). Both percentages were significantly increased compared to Pre Tx, but in the combined-therapy group, the intercourse quality $(\mathrm{Q} 3+\mathrm{Q} 4)$ improved to a greater extent than in the monotherapy group. Along with the increase in the frequency of sexual intercourse, the frequency of drug administration also increased. The 3- and 6month post-medication IIEF- 5 scores of the combined-therapy group were significantly improved compared to the scores of the monotherapy group, indicating that doxazosin may augment the effect of sildenafil. In patients with severe symptoms, this effect was more significant, and from the results of patients with severe, moderate and mild ED symptoms, we calculated that combined therapy is suitable for patients with severe and moderate symptoms; for patients with mild symptoms, either treatment is acceptable, depending on the severity of BPH. According to previously reported clinical evidence, ${ }^{16}$ for patients who failed to respond to monotherapy, a combined therapy can be administered.

It has been reported that regular treatment with PDE5 inhibitors is effective in treating LUTS secondary to BPH. ${ }^{17,18}$ Uckert et al. ${ }^{19}$ reported that PDE5 inhibitors, by interfering with cyclic nucleotidemediated pathways to elevate cyclic adenosine monophosphate and cyclic guanosine monophosphate, can reverse the tension induced by NE in isolated prostatic tissue.

Both moderate and severe patients receiving combined therapy showed significant improvements in LUTS secondary to BPH, and QoL was correlated with IPSS. For patients in the sildenafil group, after 3 and 6 months of therapy, the incidence of LUTS was not improved for moderate or severe patients, indicating that sildenafil administration on demand was not effective in treating LUTS. Particularly for patients with severe symptoms, as time passed, their symptoms were aggravated.

Sildenafil and doxazosin have been used in clinical therapies for many years. A number of patients have received each drug, and their adverse effects have been investigated comprehensively. The most commonly reported treatment-related sildenafil adverse events are headache (9\%-39\%), facial flushing (7\%-33\%), dyspepsia, dizziness, nasal congestion, abnormal vision and palpitation. Adverse events are related to the dose administered, mild in nature and self-healing by continuous use. ${ }^{20}$ Adverse effects of doxazosin during BPH therapy include dizziness, headache (10\%), acratia, oedema (2.7\%), dyspnea (2.6\%), abdominal pain (2.4\%), diarrhoea (2.3\%); dizziness and dyspnea are related to the dose administered. These effects are usually mild to moderate. ${ }^{21}$ In our study, the incidence of adverse effects was lower than reported rates, all patients showed good drug tolerance, all adverse effects were mild, and no patients left the study because of adverse effects. These results might be due to the small number of participants, the relatively strict entry criteria and the good basic health conditions of the patients. It should also be noted that at least 6 hours passed between administrations of the two drugs. From the drugs' pharmacology, we know that either of them might affect haemodynamics, especially doxazosin, with respect to blood pressure. It has been reported that tadalafil $(20 \mathrm{mg})$ could augment the 
hypotensive effects of doxazosin ( $8 \mathrm{mg}) .{ }^{22}$ However, after the 3- and 6month treatments, we found that there was no major elevation of participants' blood pressure or heart rate, which may be related to the relatively low dosage of doxazosin $(4 \mathrm{mg})$ and the shorter half-life of sildenafil compared to tadalafil.

In our study, the lack of a placebo arm ultimately limits the possible evaluation of the efficacy of the therapy under study and leads to the inability to place this trial into context with other trials. In addition, the lack of a doxazosin monotherapy arm may prevent the evaluation of the true efficacy of combined therapy on BPH.

\section{CONCLUSION}

This study indicates that the combined therapy of sildenafil and doxazosin is an effective therapeutic schedule for treating ED with LUTS secondary to $\mathrm{BPH}$, the improvement in $\mathrm{ED}$ symptoms is superior to that of sildenafil monotherapy, and the combined therapy is relatively safe.

\section{AUTHOR CONTRIBUTIONS}

ZCZ, JHL, JL, YXT, XZS, WDS and BG were mainly in charge of patients collection and follow-up. ZJ conducted statistic analysis of data and manuscript writing, and YLG and ZCX were instructors.

\section{COMPETING FINANCIAL INTERESTS}

Pfizer Inc. provided funding support for this study.

\section{ACKNOWLEDGMENTS}

This study was supported by the Investigator Initiate Research Funding from Pfizer Inc. (Cardura XL; Pfizer Inc., Dalian, China).

1 Zakaria L, Anastasiadis AG, Shabsigh R. Common conditions of the aging male: erectile dysfunction, benign prostatic hyperplasia, cardiovascular disease and depression. Int Urol Nephrol 2001; 33: 283-92.

2 Oishi K, Boyle P, Barry MJ. Epidemiology and natural history of benign prostatic hyperplasia. Proceedings of the Fourth International Consultation on $\mathrm{BPH} ; 2-5$ July 1997: Paris, France. Plymouth, UK: Health Publications, 1998, pp23-59.

3 Glina S, Santana AW, Azank F, Mello LF, Moreira ED Jr. Lower urinary tract symptoms and erectile dysfunction are highly prevalent in ageing men. BJU Int 2006; 97 763-5.

4 Rosen RC, Wei JT, Althof SE, Seftel AD, Miner M et al. Association of sexual dysfunction with lower urinary tract symptoms of BPH and BPH medical therapies: results from the BPH Registry. Urology 2009; 73: 562-6.
5 Feldman HA, Goldstein I, Hatzichristou DG, Krane RJ, McKinlay JB. Impotence and its medical and psychosocial correlates: results of the Massachusetts Male Aging Study. J Urol 1994; 151: 54-61.

6 Taylor JM, DeSouza R, Wang R. Common approach to managing lower urinary tract symptoms and erectile dysfunction. Asian J Androl 2008; 10: 45-53.

7 Lee YC, Wu WJ, Liu CC, Wang CJ, Li WM et al. The associations among eNOS G894T gene polymorphism, erectile dysfunction, and benign prostate hyperplasia-related lower urinary tract symptoms. J Sex Med 2009; 6: 3158-65.

8 Li MK, Garcia L, Patron N, Moh LC, Sundram M et al. An Asian multinational prospective observational registry of patients with benign prostatic hyperplasia, with a focus on comorbidities, lower urinary tract symptoms and sexual function. BJU Int 2008; 101: 197-202.

9 Rosen R, Altwein J, Boyle P, Kirby RS, Lukacs B et al. Lower urinary tract symptoms and male sexual dysfunction: the Multinational Survey of the Aging Male (MSAM-7) Eur Urol 2003; 44: 637-49.

10 Blanker MH, Bohnen AM, Groeneveld FP, Bernsen RM, Prins A et al. Correlates for erectile and ejaculatory dysfunction in older Dutch men: a community-based study. J Am Geriatr Soc 2001; 49: 436-42.

11 AUA Practice Guidelines Committee. AUA guideline on management of benign prostatic hyperplasia; 2003. Chapter 1: Diagnosis and treatment recommendations. J Urol 2003; 170: 530-47.

12 Uckert S, Küthe A, Jonas U. Characterization and functional relevance of cyclic nucleotide phosphodiesterase isoenzymes of the human prostate. J Urol 2001 166: 2484-90.

13 Kaplan SA, Gonzalez RR, Te AE. Combination of alfuzosin and sildenafil is superior to monotherapy in treating lower urinary tract symptoms and erectile dysfunction. Eur Urol 2008; 53: 1320

14 Oger S, Behr-Roussel D, Gorny D, Lecoz O, Lebret T et al. Combination of doxazosin and sildenafil exerts an additive relaxing effect compared with each compound alone on human cavernosal and prostatic tissue. J Sex Med 2009; 6: 836-47.

15 Oger S, Behr-Roussel D, Gorny D, Lebret T, Denoux Y et al. Combination of alfuzosin and tadalafil exerts an additive relaxant effect on human detrusor and prostatic tissues in vitro. Eur Urol 2010; 57: 699-707.

16 de Rose AF, Giglio M, Traverso P, Lantieri P. Combined oral therapy with sildenafil and doxazosin for the treatment of non-organic erectile dysfunction refractory to sildenafil monotherapy. Int J Impot Res 2002; 14: 50-3.

17 Broderick GA, Brock GB, Roehrborn CG, Watts SD, Elion-Mboussa A et al. Effects of tadalafil on lower urinary tract symptoms secondary to benign prostatic hyperplasia in men with or without erectile dysfunction. Urology 2010; 75: 1452-8.

18 Porst H, McVary KT, Montorsi F, Sutherland P, Elion-Mboussa A et al. Effects of once daily tadalafil on erectile function in men with erectile dysfunction and signs and symptoms of benign prostatic hyperplasia. Eur Urol 2009; 56: 727-35.

19 Uckert S, Sormes M, Kedia G, Scheller F, Knapp WH et al. Effects of phosphodiesterase inhibitors on tension induced by norepinephrine and accumulation of cyclic nucleotides in isolated human prostatic tissue. Urology 2008; 71: 526-30.

20 Hatzimouratidis K. Sildenafil in the treatment of erectile dysfunction: an overview of the clinical evidence. Clin Interv Aging 2006; 1: 403-14.

21 McEvoy GK, editor. American Hospital Formulary Service Drug Information 2002 Bethesda, MD: American Society of Health System Pharmacists, Inc.; 2002 (Plus Supplements). p1819.

22 Kloner RA, Jackson G, Emmick JT, Mitchell MI, Bedding A et al. Interaction between the phosphodiesterase 5 inhibitor, tadalafil and 2 alpha-blockers, doxazosin and tamsulosin in healthy normotensive men. J Urol 2004; 172 (5 Pt 1)1935-40. 